\title{
Understanding Disparities in Subpopulations of Women Who Smoke
}

\author{
Megan V. Smith • Christina Ramsay • \\ Carolyn M. Mazure
}

Published online: 21 November 2013

(C) Springer International Publishing AG 2013

\begin{abstract}
Tobacco use is the leading known cause of preventable death and disease among women. In this paper we use fundamental concepts and definitions from the general healthdisparities literature to examine smoking behavior among subpopulations of women. We focus on three factors associated with disparities in smoking behavior among subgroups of women - race and/or ethnicity, educational status, and acculturation. We suggest that research on smoking behavior among subpopulations of women is beginning to reveal not only different smoking behavior but disparities among women in different subpopulations. We conclude that subpopulationbased understanding of gender differences and disparities in smoking is critical to improvement of research design, intervention objectives, and public health policy on smoking in women.
\end{abstract}

Keywords Smoking $\cdot$ Gender differences $\cdot$ Disparities · Addiction

M. V. Smith $(\varangle) \cdot$ C. M. Mazure

Department of Psychiatry, Yale University School of Medicine,

142 Temple Street, Suite \#301, New Haven, CT 06511, USA

e-mail: megan.smith@yale.edu

C. M. Mazure

e-mail: carolyn.mazure@yale.edu

M. V. Smith • C. M. Mazure

Child Study Center, Yale University School of Medicine,

135 College Street, Suite \#220, New Haven, CT 06510, USA

M. V. Smith

Department of Epidemiology, Social \& Behavioral Sciences, Yale

School of Public Health, 135 College Street, Suite \#220, New Haven,

CT 06510, USA

C. Ramsay

Department of Psychology, Yale University, 135 College Street,

Suite \#220, New Haven, CT 06510, USA

e-mail: christina.ramsay@yale.edu

\section{Introduction}

Smoking is the single most costly health-risk behavior and the leading cause of preventable death in the United States; examination of current smoking habits shows that approximately $17 \%$ of women and $22 \%$ of men in the US smoke [1]. Smoking-cessation intervention is a critical component of tobacco-control policies, and evidence-based studies indicate it is beneficial to smokers $[2,3]$. Successful programs that target reduction of smoking are predicated on using an understanding of the different smoking behavior among subpopulations of smokers to develop and provide intervention designed for effective treatment.

For example, studies based on samples of treatment-seeking women and men find that the same proportion of women and men attempt to quit [4], and that women use even more quit strategies during an attempt than men [4] yet have greater difficulty achieving and sustaining abstinence from smoking when quitting on their own, or using first-line treatment (for example nicotine replacement therapy or pharmacologic and behavioral treatment) $[5 \cdot, 6]$. Moreover, smoking reduction requires an understanding of whether and how subpopulations of men and women are vulnerable to greater hurdles in their access to prevention strategies and treatment intervention. Numerous studies have identified different patterns of smoking behavior and disproportionate consequences of smoking not only between genders but also within gender, for example that among some racial and ethnic subpopulations of women [7, 8].

We have used fundamental concepts and definitions from the general health-disparities literature to examine smoking behavior among subpopulations of women, with a focus on three factors:

1. race and/or ethnicity;

2. educational status; and

3. acculturation. 
We suggest that research on smoking behavior among subpopulations of women is beginning to reveal not only different smoking behavior but also disparities across women in different subpopulations. We propose that application of the definitions and methodological approaches used in the general health-disparities literature to research on smoking behavior will assist in the development of intervention designed, targeted, and personalized for smoking cessation. Finally, we conclude that subpopulation-based understanding of gender differences and disparities in smoking is critical to improvement of research design, intervention objectives, and public health policy on smoking among women.

\section{Defining Health Disparities}

In 2000, the US Surgeon General's office issued several landmark reports on tobacco use, detailing dramatic differences in tobacco use and attempts to quit by subgroups, and used the term "disparities" to refer to inequities in the availability of and access to smoking-cessation services [7, 8]. The term "disparities" was specifically used to refer to the higher levels of tobacco use and lower levels of access to necessary tobacco-cessation services among racial and ethnic minority groups compared with those available to the majority white population [7].

However, definitions of health-care disparities have not been consistent in the health services research literature. For example, both the Healthy People 2010 [9] and the AHRQ National Healthcare Disparities Reports [10] provide another definition of racial and ethnic disparities as "all differences among populations in measures of health and health care". This broad definition does not take into account racial and/or ethnic group differences that many would consider appropriate in an equitable health care system, for example, different need for treatment (e.g., because of different health status) or treatment preferences. A third, more nuanced definition of disparities was coined by the Institute of Medicine (IOM). In its Unequal Treatment report [11] disparities are referred to as those "differences in health care services received by two groups that are not due to differences in the underlying health care needs or preferences of members of the groups". According to the IOM, differences attributable to the need for or preferences for services should not be regarded as constituting a disparity, but differences attributable to socioeconomic status, gender, or other factors that are based on discriminatory practices should be regarded as disparities [12].

Recently, the IOM definition of disparities has been integrated into the substance abuse treatment literature [12] and, we argue, has relevance to the conduct of research and implementation of policy on smoking behavior. The IOM definition has particular relevance to understanding disparities related to smoking in subpopulations of women, because many factors affecting whether women receive effective cessation intervention depend upon unbiased health-care systems and the conduct of research designed and implemented to develop gender-specific intervention for smoking.

\section{Applying the IOM Definition of Disparities to Subpopulations of Women Who Smoke}

Implementation of the IOM definition of health care disparities requires use of analytical methods different from those used to compare means across groups (e.g. women vs. men, African-American women vs. white women). Specifically, the IOM definition requires analytical techniques that distinguish between differences due to a given set of characteristics (defined by clinical need and patient preferences) and differences due to all other factors. Analysis is required that adjusts for number of cigarettes smoked, mental health status, and treatment preferences, and which can be used to investigate how factors such as perceived gender discrimination, educational status, race and/or ethnicity, and acculturation contribute to disparities in utilization of smoking-cessation services. One specific method is to ensure independent variables are classified into two categoriesthose to be adjusted (need) and those included in the disparity calculation (e.g. educational status, race and/or ethnicity, and acculturation).

Below we utilize the IOM definition of disparities to summarize some of the emergent results from research on disparities in smoking behavior among subpopulations of women specific to educational status, race and/or ethnicity, and acculturation. The articles we highlight utilize analytical methods and study designs that enable conclusions to be drawn about the existence and extent of disparities on the basis of the IOM definition of disparity.

\section{Disparities in Smoking Behavior and Utilization of Smoking-Cessation Treatments Among Subpopulations of Women}

Despite identification of factors associated with gender differences in smoking behavior, smoking research in the US has paid fragmented attention to subpopulations of women, focusing primarily on smoking in samples of white women seeking treatment. Consequently, our knowledge about disparities in smoking within subpopulations of women is limited. In this section, we specifically discuss disparities in access to and utilization of smoking-cessation treatment with particular focus on educational status, race and/or ethnicity, and acculturation. We conclude that, despite continuing progress in documenting the variability in smoking behavior and related consequences for subpopulations of women, much additional 
gender-specific smoking research that adheres to the IOM definition of disparities is still needed.

\section{"First and Worst"}

Health disparities researchers use the term "first and worst" to refer to the earlier disease onset and greater severity of disease often manifest among racial and ethnic minorities [13]. The "first and worst" concept is one that, although unfortunate, aptly describes disparities in smoking among women and, in particular, among subpopulations of women. Specific to women in general, a recent review of studies documenting differences between smoking by women and men [14] found that women maintain their nicotine addiction with lower levels of nicotine intake than men (e.g. "first") and are more vulnerable to the adverse health consequences of smoking, for example lung cancer (e.g. "worst") [15].

The "first and worst" concept also describes smoking behavior among African-American women in that, compared with white women, African-American women are more likely to initiate smoking at an earlier age (e.g. "first") $[16,17]$ and are more likely to be diagnosed with lung cancer when the cancer is at a more advanced stage [18], to have more aggressive forms of cancer that are resistant to treatment [19-21], and to have what are called "triple-negative tumors" (tumors that grow more quickly, and kill more frequently) (e.g. "worst") [19-21], despite being more likely to be "light" smokers (adults smoking fewer than 10 cigarettes a day) than white Americans [22]. Mortality from lung cancer is also greater among African-American women aged 35-64 than among white women of similar age [23]. In short, although the prevalence of smoking among African-American women is lower than among white women, the different ages at initiation lead to disparities in health outcomes [24, 25].

\section{The Effect of Educational Status, Race and/or Ethnicity, and Acculturation on Smoking Among Women}

Although educational status and other variables related to socioeconomic position account for many of the health disparities, race and/or ethnicity can be an added factor that is linked to poor health. In recent data from the National Survey on Drug Use and Health among adults aged 18 and older, $21 \%$ of non-Hispanic African-American women and $16 \%$ of Hispanic women reported smoking, with the highest prevalence among low income (32\%) and unemployed (41\%) women and women with less than a high school diploma (26\%) [26]. Next we elaborate on two important variables in health-disparities research-educational status and race and/or ethnicity — as separate factors affecting women's smoking behavior.

\section{Educational Status}

There is increasing evidence that education has a distinct effect on smoking behavior. Almost uniformly, findings indicate that women with little education are a particularly highrisk group. Education has affected not only the number of both young and old women who have never smoked but also the growing proportion of former smokers among highly educated women. In 2010, the prevalence of current smoking among female college graduates age 25-34 was almost 20 percentage points lower ( $9 \%$ versus approximately $30 \%$ ) than for other education groups, and the prevalence of never smoking was comparable (79\% versus $58 \%$ ) [27]. The lower the level of education, the greater the risk of being a current smoker, smoking daily, smoking heavily, being nicotine-dependent, starting to smoke at an early age, having higher levels of circulating cotinine per cigarette smoked, and continuing to smoke in pregnancy [28]. This educational disadvantage also has a pervasive negative effect on the second generation; their offspring are more likely to smoke and to have behavior problems [29].

\section{Race and/or Ethnicity}

Understanding differences and disparities in cigarette smoking requires heterogeneity in smoking patterns by gender and race and/or ethnicity to be well documented and understood, given the rapidly changing composition of the US population [30], and a growing body of research is documenting racial and/or ethnic disparities in cigarette smoking [31••]. Prevalence and type of smoking among African-American women are different from those among white women. One example is the proportion of light smokers (adults smoking fewer than 15 cigarettes a day), which is higher among African-Americans (59.2\%) than among white Americans (29.1\%) [32] and higher among AfricanAmerican women $(63 \%)$ than among African-American men $(53 \%)[33]$.

Specific to disparities in smoking cessation, racial and ethnic minority women smokers are less likely to receive advice to stop smoking, to use recommended treatments to aid cessation, or to be successful in achieving or maintaining abstinence than their white counterparts with the same frequency of smoking $[34,35]$. An exception is the implementation of home smoking restrictions, i.e. the practice of voluntarily adopting rules to limit or ban cigarette smoking in the home. Home smoking restrictions were more likely to be adopted (after an initial motivational interview encouraging their use) by African-American female smokers than by male smokers or white female smokers [33].

Within gender and broad racial and ethnic categories, there are many other groups, for example Asian-American subgroups, with diverse health needs and different barriers to 
gaining access to care for whom we have little information about access to smoking-cessation treatment [36]. Misconceptions of racial and ethnic minority populations, for example Black, Hispanic and Asian populations, as monolithic groups lacking within-group diversity, function as barriers to attempts to direct smoking-cessation efforts and could be a factor contributing to inequalities in the availability, use, and quality of treatment services among female, racial and ethnic minority groups.

Acculturation: Migration and Smoking

Among Subpopulations of Women

A comprehensive examination of gender disparities in smoking in accordance with the IOM definition should consider patterns of migration and their effect on smoking initiation and cessation between women. From other research on health disparities, we know of the powerful effect of migration on health. Almost universally across health outcomes, all racial and ethnic groups have better outcomes than their counterparts born in the United States, irrespective of gender [37]. In general, the longer an immigrant remains in the United States, the worse his or her health becomes, such that declines in health outcomes are seen with increasing generational status.

How long a population or person has resided in the US, and the extent to which US cultural norms have been adopted by the person or population, have been shown to substantially affect smoking behavior. Specific to Asian-American female smokers, acculturation seems to increase the risk of cigarette smoking [38]. In a meta-analysis that examined the prevalence of smoking among Asian women and the effect of acculturation, most commonly measured as proficiency in English, the average effect size for women was 5.26 (2.75-10.05), suggesting that acculturated Asian women are five times more likely to smoke than Asian women who maintain traditional Asian values. Among adolescents, the average effect size was 1.92 (1.223.01 ), indicating that acculturated adolescents are almost two times more likely to smoke than traditional adolescents [39].

Specific to Hispanic subpopulations, surveys indicate that Mexican-Americans smoke less than many other Hispanic subgroups including Cubans and Puerto Ricans [40] and Mexican-Americans have exceptionally low cigarette consumption, with a large fraction of smokers self-identified as "intermittent" or "occasional" rather than "daily" smokers [41]. Recent results confirm that the Hispanic advantage is not consistent among all Hispanic subgroups of women. For example, US-born Hispanic women are more likely to smoke than white women, and foreign-born Hispanic women consume many fewer cigarettes daily [42]. Smoking-related mortality for US born, non-Mexican-American Hispanic women $(24 \%)$ is higher than for white women and white men [42] with a similar smoking habit. Overall, future research must include studies of subpopulations of racial and ethnic minority women with a focus on diverse measures of acculturation including, but not limited, to language proficiency.

\section{Conclusion}

Although the prevalence of cigarette smoking among women in the US has decreased over the last three to four decades, it has emerged that race and/or ethnicity, acculturation, and educational status all substantially affect the prevalence of smoking and other smoking behavior among women [1]. We provide evidence of different smoking behavior among subpopulations of women, of disparities in access to care, and of exposure to cultural factors that affect smoking. We have also summarized major findings among subpopulations of women in accordance with the IOM definition of disparities both from national surveys and from smaller, treatment-seeking and community samples. We invoke the IOM definition of disparities because we believe it correctly separates needs or preferences for health care from the lack of access or availability of appropriate health care, and calls attention to the need for statistical methods that compare means across groups while maintaining constant the variables that determine need for smoking-cessation treatment.

Although progress continues to be made in documenting variability in smoking behavior and related consequences for subpopulations of women, much additional research is still needed. For example, large national surveys provide limited data enabling examination of factors explaining disparities in smoking behavior much beyond social demographic variables. To conform with the IOM definition of disparities, research must maintain a systematic, strong, and growing focus on subpopulations of women smokers to enable further understanding of how education, discrimination, and immigration affect observed gender differences in the extent of smoking and other comorbid conditions.

Furthermore, elucidating the extent to which observed gender differences in smoking may be because of methodological discrepancies across studies and/or environmental and psychosocial factors unique to women and specific to specific subpopulations of women has particular health relevance. For example, clarifying the methodological and contextual factors that affect smoking among subpopulations of women will facilitate future epidemiologic studies and enable the design of cessation intervention for, and policy changes directed at, specific groups of smokers - an objective conforming with the current movement toward personalized medicine.

Personalized medicine as applied to smoking-cessation intervention for women necessitates better understanding of underlying differences within and between groups of women to enable identification of characteristics of subpopulations that enable prediction of smoking cessation and help match 
smokers with a strategy that is more likely to help them quit, ultimately reducing disparities in smoking. To summarize, a more nuanced gender-specific framework is needed to examine how gender differences occur in subpopulations of women as potential disparities, and how their existence can affect the development of research and tobacco-control policies that are sensitive to the subpopulations of women most at risk.

Acknowledgments This work was supported in part by National Institutes of Health grants P50-DA033945 (ORWH, NIDA) Translational Center to Develop Gender-Sensitive Treatment for Tobacco Dependence; K12DA061050 (NIDA, NIAAA, ORWH) Building Interdisciplinary Research Careers in Women's Health; and Women's Health Research at Yale.

\section{Compliance with Ethics Guidelines}

Conflict of Interest Megan V. Smith, Christina Ramsay, and Carolyn M. Mazure declare that they have no conflict of interest.

Human and Animal Rights and Informed Consent This article does not contain any studies with human or animal subjects performed by any of the authors.

\section{References}

Papers of particular interest, published recently, have been highlighted as:

- Of importance

•- Of major importance

1. Centers for Disease Control and Prevention: Current cigarette smoking among adults - United States, 2011. MMWR 2012, 61: 889-894.

2. Fiore MC, Jaén CR, Baker TB, et al. Treating tobacco use and dependence: 2008 update. Rockville: US Department of Health and Human Services. Public Health Service; 2008.

3. Cahill K, Stevens S, Perera R, Lancaster T. Pharmacological interventions for smoking cessation: an overview and network metaanalysis. Cochrane Database Syst Rev 2013;5 CD009329.

4. Whitlock EP, Vogt TM, Hollis JF, Lichtenstein E. Does gender affect response to a brief clinic-based smoking intervention? Am J Prev Med. 1997;13:159-66.

5. Piper ME, Cook JW, Schlam TR, et al. Gender, race, and education differences in abstinence rates among participants in two randomized smoking cessation trials. Nicotine Tobacco Res. 2010;12:64757. Piper et al. examined smoking cessation rates and treatment response among 2,850 participants (57.2\% women, $11.7 \%$ Blacks, and $9.0 \%$ with less than a high school education) from two large cessation trials evaluating: nicotine patch, nicotine lozenge, bupropion, bupropion + lozenge, and nicotine patch + lozenge. Across all trials, women, blacks, and smokers with less education were found to be at elevated risk for cessation failure. The authors conclude that focused research is needed to understand the factors associated with reduced cessation in these subpopulations.

6. Brose LS, West R, McDermott MS, et al. What makes for an effective stop-smoking service? Thorax. 2011;66:924-6.

7. US Department of Health and Human Services. Tobacco use among US racial/ethnic minority groups - African Americans, American
Indians and Alaska Natives, Asian Americans and Pacific Islanders, and Hispanics: a report of the Surgeon General. Atlanta, Georgia: U.S. Department of Health and Human Services, Centers for Disease Control and Prevention, National Center for Chronic Disease Prevention and Health Promotion, Office on Smoking and Health; 1998.

8. US Department of Health and Human Services. Reducing tobacco use: a report of the Surgeon General. Atlanta, Georgia: U.S. Department of Health and Human Services, Centers for Disease Control and Prevention, National Center for Chronic Disease Prevention and Health Promotion, Office on Smoking and Health; 2000.

9. US Department of Health and Human Services. Healthy people 2010: understanding and improving health. 2nd ed. Washington, DC: U.S. Government Printing Office; 2000.

10. Agency for Healthcare Research and Quality. 2005 National Healthcare Disparities Report. 2004.

11. Institute of Medicine. In: Smedley BD, Stith AY, Nelson $\mathrm{AR}$, editors. Unequal treatment: confronting racial and ethnic disparities in health care. Washington, DC: The National Academies Press; 2003.

12. Cook BL, Alegria M. Racial-ethnic disparities in substance abuse treatment: the role of criminal history and socioeconomic status. Psychiatr Serv. 2011;62:1273-81.

13. Institute of Medicine. How far have we come in reducing health disparities?: Progress since 2000: workshop summary. Washington, DC: The National Academies Press; 2012.

14. Chilcoat HD. An overview of the emergence of disparities in smoking prevalence, cessation, and adverse consequences among women. Drug Alcohol Depend. 2009;104S:S17-23.

15. Benowitz NL. Nicotine addiction. N Engl J Med. 2010;362:2295-303.

16. Thompson AB, Moon-Howard J, Messeri PA. Smoking cessation advantage among adult initiators: does it apply to black women? Nicotine Tobacco Res. 2011;13:15-21.

17. Moon-Howard J. African American women and smoking: starting later. Am J Public Health. 2003;93:418-20.

18. Virnig BA, Baxter NN, Habermann EB, et al. A matter of race: early-versus late-stage cancer diagnosis. Health Aff. 2009;28:160-8.

19. American Cancer Society. Cancer facts \& figures for African Americans 2011-2012. Atlanta: American Cancer Society; 2011.

20. Carey LA, Perou CM, Livasy CA, et al. Race, breast cancer subtypes, and survival in the Carolina Breast Cancer Study. JAMA. 2006;295:2492-502.

21. Irvin Jr WJ, Carey LA. What is triple-negative breast cancer? Eur J Cancer. 2008;44:2799-805.

22. Mickens L, Ameringer K, Brightman M, Leventhal AM. Epidemiology, determinants, and consequences of cigarette smoking in African American women: an integrative review. Addict Behav. 2010;35:383-91.

23. US Cancer Statistics Working Group. United States Cancer Statistics: 1999-2009 Incidence and Mortality Web-based Report. Atlanta: US Department of Health and Human Services, Centers for Disease Control and Prevention and National Cancer Institute; 2013. Available at: www.cdc.gov/uscs.

24. Institute of Medicine. Reducing tobacco-related cancer incidence and mortality: workshop summary. Washington, DC: The National Academies Press; 2013.

25. Knickman JR. Institute of medicine: getting serious about the social determinants of health. Commentary. Washington, DC: The National Academies Press; 2013.

26. Centers for Disease Control and Prevention. CDC Health Disparities and Inequalities Report - United States, 2011. MMWR, 60S:109-113.

27. Maralani V. Educational inequalities in smoking: the role of initiation versus quitting. Soc Sci Med. 2013;84:129-37. 
28. Higgins ST, Chilcoat HD. Women and smoking: an interdisciplinary examination of socioeconomic influences. Drug Alcohol Depend. 2009;104S:S1-5.

29. Kandel DB, Griesler PC, Schaffran C. Educational attainment and smoking among women: risk factors and consequences for offspring. Drug Alcohol Depend. 2009;104S:S24-33.

30. Humes KR, Jones NA, Ramirez RR. Overview of race and Hispanic origin: 2010. Washington, DC: US Department of Commerce, Economics and Statistics Administration, US Census Bureau; 2011.

31.• Trinidad DR, Perez-Stable EJ, White MM, et al. A nationwide analysis of US racial/ethnic disparities in smoking behaviors, smoking cessation, and cessation-related factors. Am J Public Health. 2011;101:699-706. This study utilized national representative data to examine racial/ethnic disparities in smoking behaviors, smoking cessation, and factors associated with cessation among US adults. Based on racial and ethnic disparities found, traditional conceptualizations of cigarette addiction and the quitting process need to be revised for racial/ethnic minority smokers.

32. Novotny TE, Warner KE, Kendrick JS, Remington PL. Smoking by blacks and whites: socioeconomic and demographic differences. Am J Public Health. 1988;78:1187-9.

33. Warren JR, Okuyemi KS, Guo H, et al. Predicting home smoking restrictions among African American light smokers. Am J Health Behav. 2010;34:110-8.

34. Houston TK, Scarinci IC, Person SD, Greene PG. Patient smoking cessation advice by health care providers: the role of ethnicity, socioeconomic status, and health. Am J Public Health. 2005;93:1056-61.
35. Lopez-Quintero C, Crum RM, Neumark YD. Racial/ethnic disparities in report of physician-provided smoking cessation advice: analysis of the 2000 National Health Interview Survey. Am J Public Health. 2000;96:2235-9.

36. Murray RL, Bauld L, Hackshaw LE, McNeill A. Improving access to smoking-cessation services for disadvantaged groups: a systematic review. J Publ Health. 2009;31:258-77.

37. Landrine H, Klonoff EA. Culture change and ethnic-minority health behavior: an operant theory of acculturation. J Behav Med. 2004;27:527-55.

38. An N, Cochran SD, Mays VM, McCarthy WJ. Influence of American acculturation on cigarette smoking behaviors among Asian American subpopulations in California. Nicotine Tobacco Res. 2008;10:579-87.

39. Choi S, Rankin S, Stewart A, Oka R. Effects of acculturation on smoking behavior in Asian Americans: a meta-analysis. J Cardiovase Nurs. 2008;23:67-73.

40. Singh GK, Siahpush M. Ethnic-immigrant differentials in health behaviors, morbidity, and cause specific mortality in the United States: an analysis of two national databases. Hum Biol. 2002;74: 83-109.

41. Caraballo RS, Giovino GA, Pechacek TF, et al. Racial and ethnic differences in serum cotinine levels of cigarette smokers: Third National Health and Nutrition Examination Survey, 1988-1991. JAMA. 1998;280:135-9.

42. Fenelon A. Revisiting the Hispanic mortality advantage in the United States: the role of smoking. Soc Sci Med. 2013;82:1-9. 